Research Paper

\title{
Comparison of the Antialbuminuric Effects of L-/N-type and L-type Calcium Channel Blockers in Hypertensive Patients with Diabetes and Microalbuminuria: The Study of Assessment for Kidney Function by Urinary Micro- albumin in Randomized (SAKURA) Trial
}

\author{
Katsuayuki Ando ${ }^{\circledR}$, Kenji Ueshima², Sachiko Tanaka², Shinji Kosugi³ ${ }^{3}$, Tosiya Sato ${ }^{4}$, Hiroaki Matsuoka ${ }^{5}$ \\ Kazuwa Nakao 2,6 , Toshiro Fujita ${ }^{7}$ \\ 1. Department of Nephrology and Endocrinology, University of Tokyo Graduate School of Medicine, Tokyo, Japan \\ 2. EBM Research Center, Kyoto University Graduate School of Medicine, Kyoto, Japan \\ 3. Department of Medical Ethics/Medical Genetics, Kyoto University School of Public Health, Kyoto, Japan \\ 4. Department of Biostatistics, Kyoto University School of Public Health, Kyoto, Japan \\ 5. Utsunomiya Chuoh Hospital, Tochigi, Japan \\ 6. Department of Medicine and Clinical Science, Kyoto University Graduate School of Medicine, Kyoto, Japan \\ 7. Division of Clinical Epigenetics, Research Center for Advanced Science and Technology, University of Tokyo, Tokyo, Japan
}

$\triangle$ Corresponding author: Katsuayuki Ando, MD, Department of Nephrology and Endocrinology, University of Tokyo Graduate School of Medicine, 7-3-1 Hongo, Bunkyo-ku, Tokyo, 113-8655 Japan. Phone: +81-3-5800-9119, Fax: +81-3-5800-9119; E-Mail: katsua-tky@umin.ac.jp

(c) Ivyspring International Publisher. This is an open-access article distributed under the terms of the Creative Commons License (http://creativecommons.org/ licenses/by-nc-nd/3.0/). Reproduction is permitted for personal, noncommercial use, provided that the article is in whole, unmodified, and properly cited.

Received: 2012.11.09; Accepted: 2013.05.13; Published: 2013.07.30

\begin{abstract}
Objective: To clarify whether the L-/N-type calcium channel blocker (CCB) cilnidipine is more renoprotective than the L-type CCB amlodipine in patients with early-stage diabetic nephropathy.

Methods: In this prospective, multicenter, open-labeled, randomized trial, the antialbuminuric effects of cilnidipine and amlodipine were examined in renin-angiotensin system (RAS) inhibitor-treated patients with hypertension (blood pressure [BP]: $130-180 / 80-110 \mathrm{mmHg}$ ), type 2 diabetes, and microalbuminuria (urinary albumin to creatinine $[\mathrm{Cr}]$ ratio [UACR]: $30-300 \mathrm{mg} / \mathrm{g}$ ).

Results: Patients received cilnidipine ( $n=179$, final dose: $10.27 \pm 4.13 \mathrm{mg} /$ day) or amlodipine $(n=$ $186,4.87 \pm 2.08 \mathrm{mg} /$ day) for 12 months. Cilnidipine and amlodipine equally decreased BP. The UACR values for the cilnidipine and amlodipine groups were III $1.50 \pm 138.97$ and $88.29 \pm 63.45$ $\mathrm{mg} / \mathrm{g}$, respectively, before treatment and $107.93 \pm 130.23$ and $89.07 \pm 97.55 \mathrm{mg} / \mathrm{g}$, respectively, after treatment. The groups showed similar changes for the natural logarithm of the UACR, serum $\mathrm{Cr}$, and estimated glomerular filtration rate.

Conclusions: Cilnidipine did not offer greater renoprotection than amlodipine in RAS inhibitor-treated hypertensive patients with type 2 diabetes and microalbuminuria.
\end{abstract}

Key words: L-/N-type Calcium Channel Blocker, Urinary Albumin, Diabetic Nephropathy, Hypertension, Renin-Angiotensin System Inhibitor

\section{INTRODUCTION}

Considerable clinical evidence suggests that renin-angiotensin system (RAS) inhibitors are benefi- cial as first-line antihypertensive agents for hypertension in patients with diabetic or nondiabetic 
nephropathy [1-4]. However, by themselves, RAS inhibitors are unable to maintain the blood pressure (BP) at a level below $130 / 80 \mathrm{mmHg}$, as is required in patients with chronic kidney disease (CKD). For such BP maintenance, second-line depressor agents are required. Dihydropyridine-type calcium channel blockers (CCBs) are frequently used in combination with RAS inhibitors in hypertensive patients with CKD because of their strong BP-lowering properties and minimal adverse side effects [5]. However, CCBs are not always able to protect against kidney injury, as was shown in the Renoprotection in Patients with Nondiabetic Chronic Renal Disease (REIN)-2 [6]. And, in Gauging Albuminuria Reduction with Lotrel in Diabetic Patients with Hypertension (GUARD) [7] trials, the antialbuminuric effect of CCB was weaker than that of diuretics in RAS inhibitor-treated hypertensive patients with type 2 diabetic nephropathy.

The uncertain renoprotective effects of L-type CCBs may be due to the presence of L-type calcium channels at the afferent but not efferent arterioles [8]. L-type CCBs cause afferent arteriole-specific vasodilation, which increases the glomerular pressure. This adverse action of L-type CCBs in the glomerular microcirculation counteracts their ability to attenuate glomerular hypertension through the systemic decrease in BP. Thus, the use of L-type CCBs is not always beneficial in patients with renal dysfunction.

On the other hand, the Cilnidipine versus Amlodipine Randomized Trial for Evaluation in Renal Disease (CARTER) study [9] recently demonstrated that the L-/N-type CCB cilnidipine, but not the L-type CCB amlodipine, decreased urinary protein levels in RAS inhibitor-treated hypertensive patients with macroproteinuria. The N-type calcium channel exists at the peripheral sympathetic nerve endings, and its inhibition suppresses norepinephrine release from nerve terminals. Cilnidipine has been demonstrated to inhibit sympathetic nerve activity [10-12]. Because both the afferent and efferent arterioles of the glomerulus are sympathetically innervated, cilnidipine ameliorates glomerular hypertension through the vasodilation of both arterioles $[13,14]$. Thus, cilnidipine has an antiproteinuric effect in CKD patients, who have increased sympathetic activity $[15,16]$.

However, the CARTER study found that the antiproteinuric effect of cilnidipine did not significantly differ from that of amlodipine in the diabetic subgroup of patients with macroproteinuria [9]. Diabetic subjects with advanced nephropathy (macroproteinuria) also frequently have diabetic neuropathy. In such patients, cilnidipine may be unable to exert an effective renoprotective effect through renal sympathoinhibition. On the other hand, cilnidipine might be renoprotective in the early stage of diabetic nephrop- athy $(\mathrm{DN})$, when renal nerve function is still intact.

To clarify whether the L-/N-type CCB cilnidipine is more renoprotective than the L-type CCB amlodipine in patients with early-stage DN, we compared the antialbuminuric effects of cilnidipine and amlodipine in RAS inhibitor-treated diabetic patients with microalbuminuria.

\section{PATIENTS \& METHODS}

A prospective, multicenter, open-labeled, randomized trial, the Study of Assessment for Kidney Function by Urinary Microalbumin in Randomized (SAKURA) trial, was performed in 77 clinics and hospitals in Japan (17). The trial was registered with the University Hospital Medical Information Network-Clinical Trials Registry (UMIN-CTR) under trial identification number UMIN000001247. It was approved by the Institutional Review Board of the University of Tokyo Clinical Research Center (reference number P2007028-11X) and by the review boards of all other concerned medical facilities. The implementation and data management of the trial were done by the executive office at the EBM Research Center of the Kyoto University Graduate School of Medicine. The trial was conducted in accordance with the principles of the Declaration of Helsinki. Written informed consent was obtained from all participating patients after they had received oral and written explanations about the trial.

\section{Participants}

Patients with hypertension (outpatient systolic/diastolic $\mathrm{BP} \geq 130 / 80$ and $<180 / 110 \mathrm{mmHg}$ ) with type-2 diabetes and microalbuminuria (urinary albumin to creatinine $[\mathrm{Cr}]$ ratio $[\mathrm{UACR}] \geq 30$ and $<300$ $\mathrm{mg} / \mathrm{g}$ in spot urine) who were being treated with a RAS inhibitor (angiotensin receptor blocker [ARB] or angiotensin-converting enzyme [ACE] inhibitor) were recruited for this study. The detailed inclusion and exclusion criteria were reported previously [17]. The required sample size (438 patients [17]) was estimated according to previous reports $[18,19]$ as the size needed for the detection of a significant difference when the difference in the primary endpoint between the two arms was $23 \%$, with an alpha error of $5 \%$ and a power of $90 \%$.

\section{Interventions}

Eligible study subjects were randomly allocated to two groups and treated with cilnidipine (started at $10 \mathrm{mg} /$ day, then adjusted to $5-20 \mathrm{mg} /$ day) or amlodipine (started at $5 \mathrm{mg} /$ day, then adjusted to $2.5-10$ $\mathrm{mg} /$ day). The target BP was $<130 / 80 \mathrm{mmHg}$. If cilnidipine or amlodipine combined with a RAS inhibitor failed to reduce the BP to the target level, then addi- 
tional antihypertensive drugs (other than a RAS inhibitor or a CCB) were administered. The treatment period was 1 year.

\section{Outcome measures}

The primary endpoint was the change in the natural logarithm of the UACR $(\mathrm{mg} / \mathrm{g})$ from the baseline (average of two consecutive measurements during a 4-week period before treatment) to the endpoint (after 12 months of treatment). Laboratory tests were performed at a central laboratory (Mitsubishi Chemical Medicine Inc, Tokyo). The urinary albumin level was measured by the Bromcresol green photometric method (IatroFine ALB II), and Cr was measured with an enzymatic colorimetric assay (IatroLQ CRE(A) II).

Secondary outcomes were the absolute value of the UACR (mg/g), CKD stage [20], serum Cr level, estimated glomerular filtration rate (eGFR) calculated with the "Modified Diet in Renal Disease (MDRD) formula" modified by the Japanese Society of Nephrology [21], cardiovascular events [17], BP levels, and pulse rate $(\mathrm{PR})$.

\section{Statistical Analysis}

Data were analyzed in the full analysis set. Subjects who did not meet eligibility criteria, who were not administered the assigned drugs (cilnidipine or amlodipine), or who had no data after the randomized treatment assignments were not included in the analysis. Data were expressed as the mean \pm standard deviation (s.d.) and percentage. The safety of the treatment assignments was assessed in a safety analysis set.

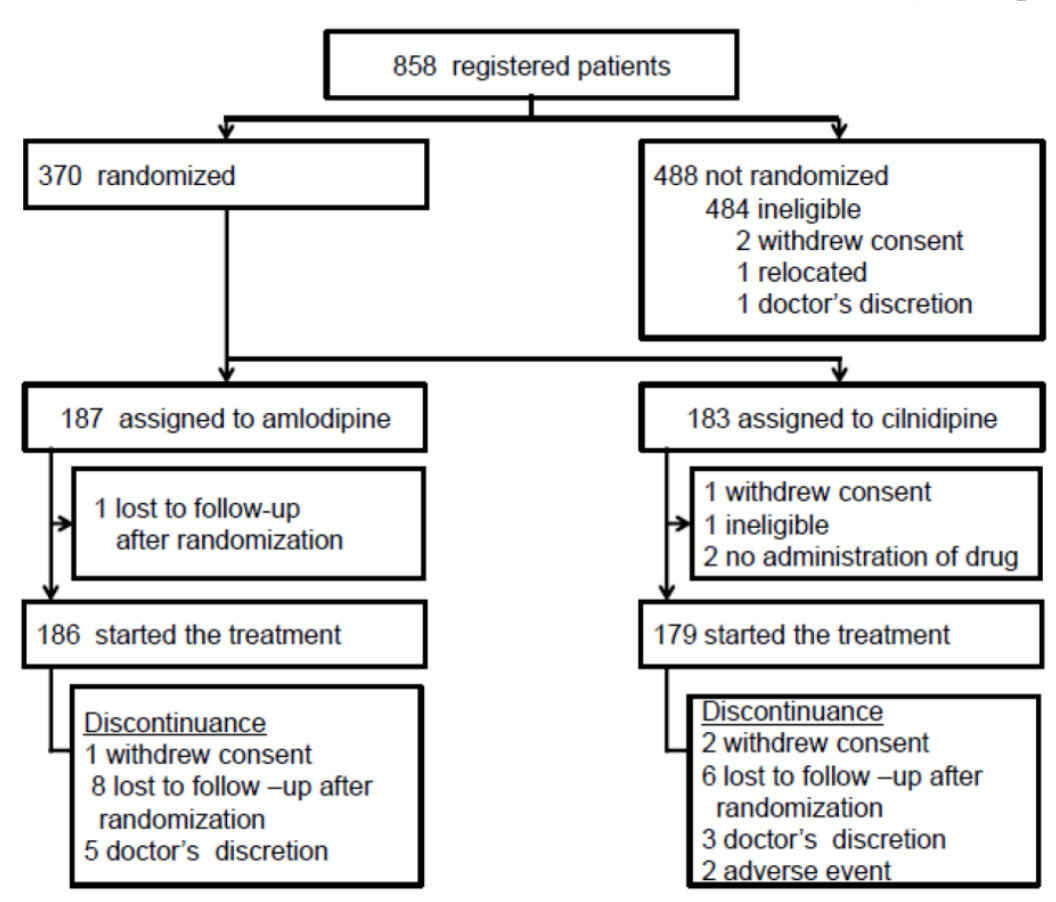

For the primary analysis, changes in the natural logarithm of the UACR from the baseline (before treatment) to endpoint (after 12 months of treatment) were compared between the two arms by using the analysis of covariance with stratification factors (UACR, systolic BP, and hemoglobin A1c [HbA1c]) [15] as covariates. For the secondary endpoints, the analysis of covariance was used for continuous data, and the chi-squared test was used for categorical data. The statistical significance level was set at $P \leq 0.05$. Statistical analysis was performed with SAS version 9.2 (SAS Institute Inc., NC).

Subgroup analysis was performed according to the following parameters: age ( $\geq 65$ or $<65$ years), sex (male or female), dyslipidemia or organ damage (existence or nonexistence), body mass index (BMI $\geq 25$ or $<25 \mathrm{~kg} / \mathrm{m}^{2}$ ), left ventricular hypertrophy ( $\mathrm{LVH}$; Sv1 + Rv5 $\geq 3.5 \mathrm{mV}$ by electrocardiography, existence or nonexistence), PR ( $\geq 75$ or $<75$ beats/min $[\mathrm{bpm}])$, serum Cr $(\geq 0.75$ or $<0.75 \mathrm{mg} / \mathrm{dL}), \mathrm{UACR}(\geq 75$ or $<$ $75 \mathrm{mg} / \mathrm{g}), \mathrm{HbA1c}(\geq 7 \%$ or $<7 \%)$, eGFR $(\geq 60$ or $<60$ $\left.\mathrm{mL} / \mathrm{min} / 1.73 \mathrm{~m}^{2}\right)$, systolic BP before treatment $(\geq 130$ or $<130 \mathrm{mmHg})$, diastolic BP before treatment $(\geq 80$ or $<80 \mathrm{mmHg}$ ), and attainment of target BP after treatment (systolic BP < $130 \mathrm{mmHg}$ and diastolic BP < $80 \mathrm{mmHg}$, attainment or nonattainment).

\section{RESULTS}

The full analysis set included 365 patients (cilnidipine arm: $n=179$, amlodipine arm: $n=186$ ) (Figure 1). The follow-up of two patients (cilnidipine arm: $n=$ 2, amlodipine arm: $n=0$ ) was discontinued due to adverse events, and the follow-up of 25 patients (cilnidipine arm: $n=11$, amlodipine arm: $n=14$ ) was discontinued for other reasons. Most of the baseline data were well balanced between the two groups, although body weight $(P$ $=0.037)$ and BMI $(P=0.040)$ were greater in the cilnidipine group (Table 1, 2) (17). The baseline UACR was higher in the cilnidipine group than in the amlodipine group (Table 3: $P=0.040$ ). However, the median UACR was almost the same in both groups (67.4 vs. $63.5 \mathrm{mg} / \mathrm{g}$ ), and the average natural logarithm of the UACR was approximately the same between the groups $(P=0.224)$.

Figure I. Flowchart for patient enrollment in the SAKURA study 
Table I. Patient characteristics

\begin{tabular}{llll}
\hline Parameter & Total & Cilnidipine & Amlodipine \\
\hline $\mathrm{N}$ & 365 & 179 & 186 \\
Male & $240(65.8 \%)$ & $112(62.6 \%)$ & $128(68.8 \%)$ \\
Age (years) & $63.84 \pm 8.46$ & $63.27 \pm 8.72$ & $64.39 \pm 8.19$ \\
Height $(\mathrm{cm})$ & $160.55 \pm 9.07$ & $160.86 \pm 9.49$ & $160.40 \pm 8.70$ \\
Body weight $(\mathrm{kg})$ & $66.87 \pm 12.93$ & $68.31 \pm 14.52$ & $65.48 \pm 11.05$ \\
BMI (m $\left.{ }^{2} / \mathrm{kg}\right)$ & $25.84 \pm 3.91$ & $26.27 \pm 4.34$ & $25.42 \pm 3.41$ \\
Dyslipidemia & $179(49.0 \%)$ & $87(48.6 \%)$ & $92(49.5 \%)$ \\
Organ damage & $55(15.1 \%)$ & $32(17.9 \%)$ & $23(12.4 \%)$ \\
Cerebrovascular & $20(5.5 \%)$ & $11(6.1 \%)$ & $9(4.8 \%)$ \\
$\quad$ disease & $49(13.4 \%)$ & $26(14.5 \%)$ & $23(12.4 \%)$ \\
Diabetic neuropathy & $39(10.7 \%)$ & $22(12.3 \%)$ & $17(9.1 \%)$ \\
Diabetic retinopathy & $12(3.3 \%)$ & $6(3.4 \%)$ & $6(3.2 \%)$ \\
Myocardial infarc- & $13(3.6 \%)$ & $8(4.5 \%)$ & $5(2.7 \%)$ \\
$\quad$ tion & $7(1.9 \%)$ & $4(2.2 \%)$ & $3(1.6 \%)$ \\
Angina pectoris & $9(2.5 \%)$ & $5(2.8 \%)$ & $4(2.2 \%)$ \\
$\quad$ Heart failure & & & \\
Arteriosclerosis & & & \\
$\quad$ obliterans & & &
\end{tabular}

Data are shown as the no. of patients (\%) or the mean \pm SD. Abbreviations: BMI: body mass index
Table 2. Baseline data

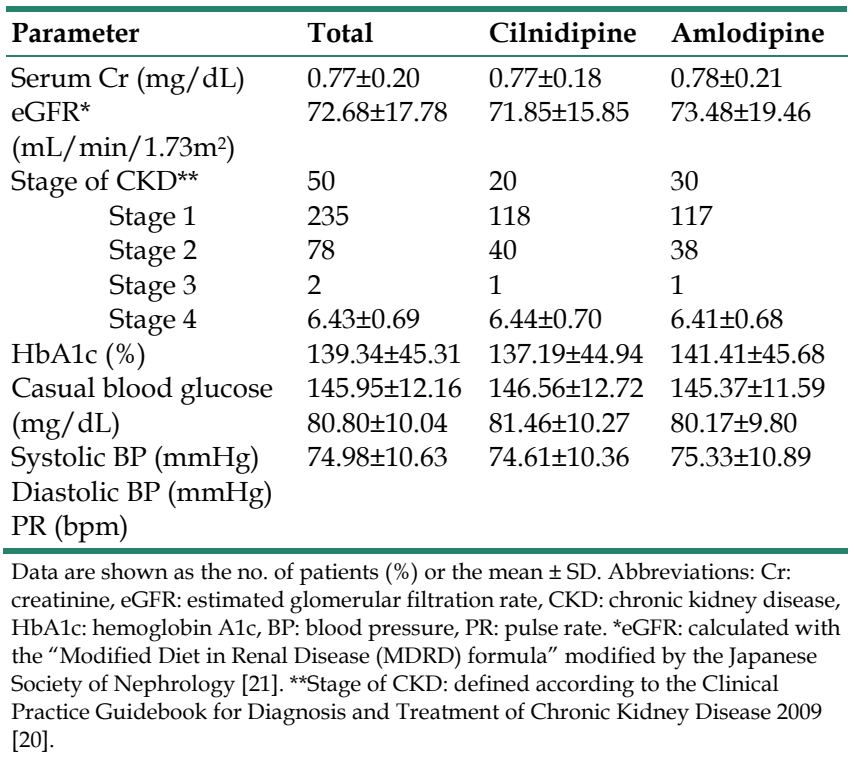

Table 3. UACR* at 3, 6, 9, and 12 months of treatment

\begin{tabular}{|c|c|c|c|c|c|c|c|}
\hline Time-point & $\mathbf{N}$ & Total & $\mathbf{N}$ & Cilnidipine & $\mathbf{N}$ & Amlodipine & \\
\hline Baseline & 365 & $99.67 \pm 107.82$ & 179 & $111.50 \pm 138.97$ & 186 & $88.29 \pm 63.45$ & \\
\hline log transformed & & $4.32 \pm 0.69$ & & $4.37 \pm 0.76$ & & $4.28 \pm 0.62$ & \\
\hline 3 months & 345 & $80.32 \pm 97.93$ & 170 & $85.05 \pm 114.06$ & 175 & $75.73 \pm 79.25$ & \\
\hline log transformed & & $3.94 \pm 0.91$ & & $3.96 \pm 0.93$ & & $3.92 \pm 0.88$ & \\
\hline 6 months & 330 & $80.09 \pm 93.86$ & 162 & $81.71 \pm 95.95$ & 168 & $78.53 \pm 92.06$ & \\
\hline log transformed & & $3.94 \pm 0.91$ & & $3.94 \pm 0.93$ & & $3.94 \pm 0.89$ & \\
\hline 9 months & 319 & $91.33 \pm 96.22$ & 158 & $97.67 \pm 101.55$ & 161 & $85.10 \pm 90.57$ & \\
\hline log transformed & & $4.10 \pm 0.90$ & & $4.15 \pm 0.93$ & & $4.05 \pm 0.87$ & \\
\hline 12 months & 330 & $98.38 \pm 115.07$ & 163 & $107.93 \pm 130.23$ & 167 & $89.07 \pm 97.55$ & \\
\hline log transformed & & $4.12 \pm 0.95$ & & $4.16 \pm 1.02$ & & $4.08 \pm 0.88$ & \\
\hline \multicolumn{8}{|c|}{ The change in the natural logarithm from baseline } \\
\hline Time-point & $\mathbf{N}$ & Total & $\mathbf{N}$ & Cilnidipine & $\mathbf{N}$ & Amlodipine & p-value ** \\
\hline 3 months & 345 & $-0.38 \pm 0.74$ & 170 & $-0.40 \pm 0.76$ & 175 & $-0.35 \pm 0.72$ & 0.586 \\
\hline 6 months & 330 & $-0.38 \pm 0.78$ & 162 & $-0.42 \pm 0.83$ & 168 & $-0.34 \pm 0.73$ & 0.441 \\
\hline 9 months & 319 & $-0.23 \pm 0.74$ & 158 & $-0.21 \pm 0.77$ & 161 & $-0.24 \pm 0.70$ & 0.764 \\
\hline 12 months & 330 & $-0.21 \pm 0.78$ & 163 & $-0.21 \pm 0.86$ & 167 & $-0.21 \pm 0.69$ & 0.96 \\
\hline
\end{tabular}

* UACR: Urinary albumin to creatinine ratio. Data are expressed as $\mathrm{mg} / \mathrm{g}$. ** Analysis of covariance considering stratification factors of randomization was used for examining the difference means of the change in the log-transformed UACR at each time point. Multiplicity was not considered.

The final doses of cilnidipine and amlodipine were $10.27 \pm 4.13$ and $4.87 \pm 2.08 \mathrm{mg} /$ day, respectively. The frequency of ARB or ACE inhibitor administration was the same for the two groups (Table 4). Both cilnidipine (systolic and diastolic BP, after treatment: $130.40 \pm 13.93 / 73.37 \pm 10.20 \mathrm{mmHg}$ ) and amlodipine (129.65 \pm 13.33/71.75 $\pm 9.79 \mathrm{mmHg})$ equally decreased BP (Figure 2), and the changes were not different between the groups (systolic and diastolic BP: $P=0.88$ and $P=0.51$, respectively). The PR was unaffected by either drug (after treatment: $74.19 \pm 11.96$ and $74.19 \pm 11.63 \mathrm{bpm})$, and the change was not significant between the two groups $(P=0.46)$.
The UACR was seemingly decreased after 3 or 6 months of treatment; In cilnidipine group, UACR seems to be decreased largely to $85.05 \mathrm{mg} / \mathrm{g}(-23.72 \%$ reduction compared to baseline) in 3 months, 81.71 $\mathrm{mg} / \mathrm{g}(-26.72 \%$ reduction) in 6 months, whereas in amlodipine group, UACR decreased to $75.73 \mathrm{mg} / \mathrm{g}$ $(-14.23 \%$ reduction) in 3 months, $78.53 \mathrm{mg} / \mathrm{g}(-11.05 \%$ reduction) in 6 months. However, UACR tended to return to the baseline value after 12 months of treatment with either drug (Table 3). Nevertheless, analysis of log-transformed UACR does not suggest the significant difference of changes in UACR between the two arms. The change in the natural logarithm of 
the UACR after 12 months of treatment was $-0.21 \pm$ 0.69 in the cilnidipine group and $-0.21 \pm 0.86$ in the amlodipine group. The difference between the groups was estimated to be 0.00 ( $95 \%$ confidence interval: -0.16 to $0.17, P=0.96$ ). Thus, cilnidipine and amlodipine had similar effects on UACR in hypertensive patients with diabetic microalbuminuria.

Table 4. Pretreatment medications

\begin{tabular}{clll}
\hline Medication & Total & Cilnidipine & Amlodipine \\
\hline Antihypertensive & & & \\
ARB & $320(87.7 \%)$ & $159(88.8 \%)$ & $161(86.6 \%)$ \\
ACE inhibitor & $62(17.0 \%)$ & $25(14.0 \%)$ & $37(19.9 \%)$ \\
Diuretic & $35(9.6 \%)$ & $20(11.2 \%)$ & $15(8.1 \%)$ \\
Alpha blocker & $9(2.5 \%)$ & $3(1.7 \%)$ & $6(3.2 \%)$ \\
Beta blocker & $19(5.2 \%)$ & $9(5.0 \%)$ & $10(5.4 \%)$ \\
Statin & $50(13.7 \%)$ & $26(14.5 \%)$ & $24(12.9 \%)$ \\
\hline
\end{tabular}

ARB: angiotensin receptor blocker, ACE: angiotensin-converting enzyme

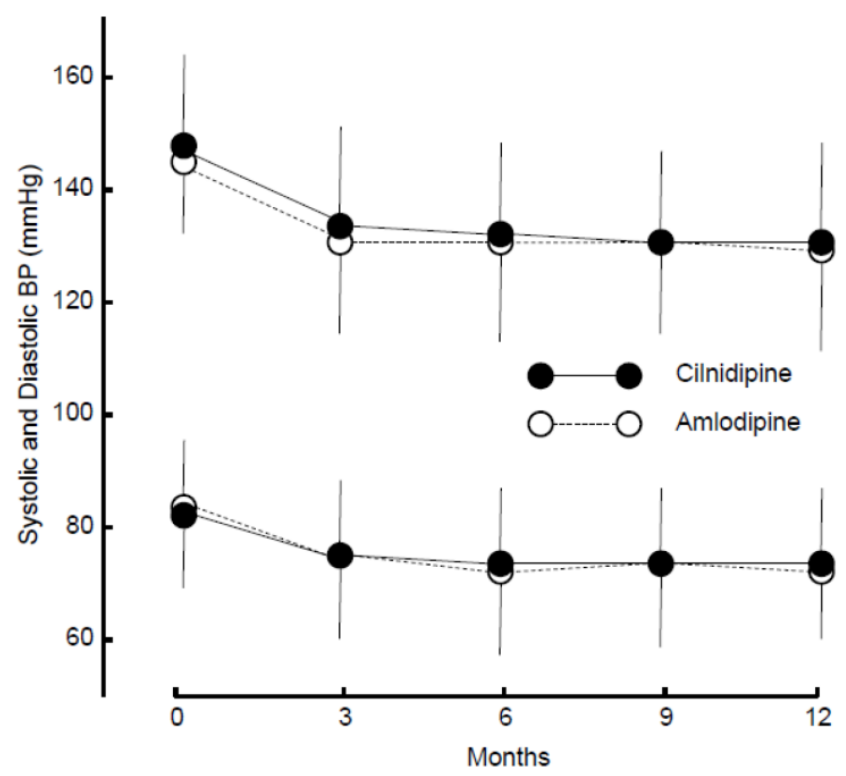

Figure 2. Changes in systolic and diastolic blood pressure (BP). Average and s.d. of systolic and diastolic BP are shown.

No changes were observed in the serum $\mathrm{Cr}$ level (cilnidipine: $0.79 \pm 0.22$, amlodipine: $0.81 \pm 0.24$ $\mathrm{mg} / \mathrm{dL}$ ) or eGFR level (cilnidipine: $71.07 \pm 17.96$, amlodipine: $\left.70.89 \pm 20.56 \mathrm{~mL} / \mathrm{min} / 1.73 \mathrm{~m}^{2}\right)$ with treatment. Changes in these two parameters were not different between the two groups $(P=0.31$ and $P=0.13$, respectively).

The CKD stage was unchanged in 96 patients, advanced in 20 patients, and regressed in 26 patients after treatment with cilnidipine, and was unchanged in 89 patients, advanced in 21 patients, and regressed in 34 patients after treatment with amlodipine. The distribution of CKD stages was not different between the two groups before and after treatment $(P=0.94)$. Cardiovascular events occurred in one patient in the cilnidipine group (stroke: $n=1$ ) and two patients in the amlodipine group (stroke: $n=1$, myocardial infarction: $n=1)$. The incidence of cardiovascular events was not different between the two groups $(P=0.58)$.

Subgroup analysis according to any stratification factor showed no difference in the change in the natural logarithm of the UACR between the two groups. In the subgroup that attained the target $\mathrm{BP}$ with treatment, the UACR was decreased in the cilnidipine arm (from $142.36 \pm 200.50$ to $96.46 \pm 107.62$ $\mathrm{mg} / \mathrm{g}$ ) but not in the amlodipine arm (from $83.08 \pm$ 58.28 to $78.52 \pm 92.00 \mathrm{mg} / \mathrm{g}$ ), although the changes were not significantly different between the two groups (changes in the natural logarithm: $-0.37 \pm 0.83$ vs. $-0.26 \pm 0.68, P=0.524)$. In the subgroup that did not attain the target BP, the UACR was not decreased in either treatment arm.

Similar numbers of non-severe adverse events were observed in the cilnidipine group $(n=14)$ and the amlodipine group $(n=10)$ (Table 5). Two severe adverse events (stroke: $n=1$, interstitial pneumonia: $n$ $=1$ ) occurred in the cilnidipine group, and five severe events (colon carcinoma: $n=2$, stroke: $n=1$, acute pancreatitis, $n=1$, and myocardial infarction, $n=1$ ) occurred in the amlodipine group.

Table 5. Adverse events

\begin{tabular}{lll}
\hline & Cilnidipine & Amlodipine \\
\hline Non-severe adverse events & 14 & 10 \\
Dizziness & 0 & 2 \\
Breathing trouble & 1 & 0 \\
Nausea/vomiting & 0 & 1 \\
Edema & 0 & 1 \\
Depression & 1 & 0 \\
Appetite loss & 1 & 0 \\
Subcutaneous bleeding & 0 & 1 \\
Complete AV block & 0 & 1 \\
Erythropenia & 2 & 0 \\
Increased BUN & 0 & 1 \\
Increased Cr & 1 & 1 \\
Increased triglyceride & 3 & 0 \\
Increased uric acid & 1 & 0 \\
Increased fasting BS & 1 & 0 \\
Dyspotassemia & 3 & 1 \\
Other & 0 & 1 \\
Severe adverse Events & 2 & 5 \\
Stroke & 1 & 1 \\
Myocardial Infarction & 0 & 1 \\
Carcinoma & 0 & 2 \\
Acute pancreatitis & 0 & 1 \\
Interstitial pneumonia & 1 & 0 \\
\hline Abbrevions: AV: atio-ver & 0 & 65
\end{tabular}

Abbreviations: AV: atrio-ventricular, BUN: blood urea nitrogen, BS: blood sugar. See abbreviations in Table 2. 


\section{DISCUSSION}

According to the data of the present SAKURA trial, L-/N-type CCB cilnidipine did not result in a greater antialbuminuric effect than L-type CCB amlodipine in RAS inhibitor-treated hypertensive patients with diabetes and microalbuminuria. This finding is similar to an observation made previously in diabetic patients with macroproteinuria in the CARTER study [9], although renal nerve function is thought to be less damaged in diabetic microalbuminuria than it is in diabetic macroproteinuria. In addition to the CARTER study [9], many small-sized studies (i.e., with 28 to 50 patients) [22-24] in nondiabetic patients with CKD and microalbuminuria have shown that cilnidipine has a greater antialbuminuric effect than amlodipine. In addition, Konoshita et al. recently showed in a crossover study that the antialbuminuric effect of cilnidipine was greater than that of amlodipine in a relatively large number $(n=110)$ of hypertensive patients, approximately $20 \%$ of whom had diabetes [25].

The antialbuminuric and renoprotective effects of L-/N-type CCBs are at least partially due to the amelioration of glomerular hypertension through efferent arteriolar vasodilation, which L-/N-type CCBs achieve via their sympatholytic effect. Sympathoactivation is thought to play an important role in the acceleration of kidney injury [26]. However, in previous reports, sympathetic dysfunction did not correlate with urinary protein levels or $\mathrm{Cr}$ clearance in diabetic patients with urinary protein levels $\geq 300$ $\mathrm{mg} /$ day (or urinary albumin levels $\geq 100 \mathrm{mg}$ /day) [27]. This finding is compatible with the results in the present study and in the diabetic subgroup analysis in the CARTER study [9].

In diabetes, the main mechanisms of glomerular hyperfiltration (which may underlie the initiation and progression of $\mathrm{DN}$ ) are by increases in the levels of hormones, such as insulin-like growth factor 1 [28], atrial natriuretic peptide [29], intracellular accumulation of sorbitol and protein glycosylation [30], and activated tubuloglomerular feedback, which are caused by increased tubular sodium reabsorption through hyperinsulinemia and hyperglycemia. Sympathetic nerve activation is not thought to be a major mechanism of glomerular hyperfiltration in DN [31]. The lack of a clear difference in the antialbuminuric effects of cilnidipine and amlodipine in the present study may be due to the marginal contribution of sympathetic nerve activation to the progression of DN.

Only a few small-scale clinical trials have compared the antialbuminuric effects of cilnidipine to those of L-type CCBs in early-stage DN. A small-sized
( $n=28)$, short-duration (3 months) study [32] showed that switching from amlodipine to cilnidipine decreased urinary albumin levels in patients with DN and microalbuminuria. Another small-sized $(n=35)$ crossover study [33] suggested the cilnidipine was superior to amlodipine for the treatment of hypertensive patients with type 2 diabetes. Cilnidipine showed a greater antialbuminuric effect than L-type CCBs (amlodipine and slow-acting nifedipine) in diabetic hypertensive patients with microalbuminuria $(n=69$, crossover study) [34]. However, in diabetic patients, cilnidipine had less of an antiproteinuric effect than the L-/T-type CCB benidipine, which has also been suggested as a renoprotective CCB [35] (subgroup analysis with $n=112$ [36] and small-sized study [37] with $n=40$ ). However, the effects of cilnidipine and benidipine were almost the same in nondiabetic subjects [36]. Thus, the antiproteinuric effect of cilnidipine may be weaker in diabetic patients than in nondiabetic patients with CKD.

Even if sympathetic activation plays only a minor role in glomerular hyperfiltration in $\mathrm{DN}$, it is difficult to understand why cilnidipine, which inhibits sympathetic nerve function, an overall important element in glomerular microcirculation, did not reduce albuminuria more substantially than amlodipine. In previous studies, chronic renal denervation normalized the increases in GFR and glomerular volume in rats with streptozotocin-induced diabetes [38], and the sympatholytic agent moxonidine reduced microalbuminuria in a small number $(n=15)$ of patients with type $1 \mathrm{DN}$ (microalbuminuria) [39]. The differences between the results obtained with cilnidipine in the present study and those obtained with renal denervation and moxonidpine treatment in previous studies may be due to the weaker effects the former treatments than the latter ones on sympatholytic activity. In pithed rats, the pressor response of sympathetic nerve stimulation was suppressed by cilnidipine; however, the effect of cilnidipine was apparently weaker than that of N-type CCB omega-conotoxin [10]. Omega-conotoxin showed bradycardic action in anesthetized rats, whereas cilnidipine did not [10]. Thus, the sympatholytic action of cilnidipine, although mild enough to protect the nondiabetic kidney from injury, may be too weak to counteract the glomerular hyperfiltration in the diabetic kidney caused by huge afferent arteriolar vasodilation. Alternatively, the use of a more appropriate therapy that lowers the BP (amlodipine group in the present and CARTER [9] studies; $129.65 \pm 13.33 / 71.75$ \pm 9.79 and $134.5 \pm 16.6 / 77.9 \pm 9.4 \mathrm{mmHg}$ ) may suppress the progression of kidney damage and blunt the antialbuminuric superiority of cilnidipine.

The present study had some limitations. First, 
the sample size calculation was based on two studies: a study that examined the antialbuminuric effect of cilnidipine in valsartan-treated patients with diabetes and microalbuminuria [18], and a study which examined the antialbuminuric effect of amlodipine in fosinopril-treated patients with diabetes and microalbuminuria [19]. These studies were used for the calculations because, when the protocol was designed (in 2007), no trials of head-to-head comparisons between cilnidipine and L-type CCBs in DN were available. The actual statistical power of the study was considered to be sufficient, although the sample size was slightly smaller than the estimated sample size. However, we cannot deny the possibility that study population was too small to be compared.

Second, although similar UACR levels at the first urinalysis (cilnidipine: $99.6 \pm 70.5$, amlodipine: $88.5 \pm$ $55.9 \mathrm{mg} / \mathrm{g}$ ) were attained for randomization as a stratified factor, the baseline data, which were averaged from two UACR data points, were higher in the cilnidipine group (Table 3) [17]. However, the two groups showed similar UACR medians and similar average natural logarithms of the UACR. Therefore, the baseline values of UACR might not affect the evaluation of the primary endpoint, although the variability of UACR was different between the two groups. The UACR levels may have been too variable to detect the relatively small differences between the antialbuminuric effects of cilnidipine and those of amlodipine.

Third, the patients in the two groups demonstrated different average body weights and BMIs (Table 1). These differences might have affected the response of UACR to antihypertensive drugs because obesity is known to accelerate albuminuria [40,41]. However, subgroup analysis using BMI values did not show that the two drugs had different effects. Subgroup analysis using attainment of the target BP as a criterion showed a greater antialbuminuric effect for cilnidipine, although the difference was not significant. Thus, in selected cases of DN, cilnidipine might help protect against kidney injury.

Forth, there were a few patients with diabetic neuropathy $(14.5 \%$ [ $n=26]$ and $12.4 \%$ [ $n=23]$ of cilnidipine and amlodipine groups). The diagnosis of diabetic neuropathy was done by the presence or absence of its symptoms. Thus, some study subjects, who were diagnosed as free from diabetic neuropathy, might have a mild to moderate neuropathy, which masked the antialbuminuric effects of the sympatholytic CCB. Thus, the sympatholytic CCB cilnidipine might be beneficial in patients with very early stage of $\mathrm{DN}$ or hyperfiltrated kidney (prenephropathy), although the present study cannot clarify this possibility.
In conclusion, the present study did not find that L-/N-type CCB cilnidipine was superior to L-type $\mathrm{CCB}$ amlodipine for the treatment of albuminuria in hypertensive patients with early-stage $\mathrm{DN}$, although cilnidipine may decrease the urinary protein or albumin level more substantially than L-type CCBs in hypertensive patients with nondiabetic CKD [9,22-24]. The different effects of L-/N-type CCBs might be due to pathophysiological differences between diabetic and nondiabetic nephropathies. Further studies are required to determine whether the antialbuminuric effects of cilnidipine are superior to those of amlodipine in $\mathrm{DN}$.

\section{ACKNOWLEDGMENTS}

The SAKURA trial was funded by the EBM Research Center of the Kyoto University Graduate School of Medicine with an unrestricted grant from Mochida Pharmaceutical Co., LTD and Ajinomoto Pharmaceuticals Co., LTD.

\section{Competing Interests}

The authors have declared that no competing interest exists.

\section{References}

1. Ando K, Fujita T: Anti-diabetic effect of blockade of the renin-angiotensin system. Diab Obes Metab 2006;8:396-403.

2. Ogihara T, Kikuchi K, Matsuoka H, et al; The Japanese Society of Hypertension Committee: The Japanese Society of Hypertension Guidelines for the Management of Hypertension (JSH 2009). Hypertens Res 2009;32:3-107.

3. Mancia G, De Backer G, Dominiczak A, et al; Management of Arterial Hypertension of the European Society of Hypertension; European Society of Cardiology. 2007 Guidelines for the Management of Arterial Hypertension. The Task Force for the Management of Arterial Hypertension of the European Society of Hypertension (ESH) and of the European Society of Cardiology (ESC). 2007 Guidelines for the Management of Arterial Hypertension. The Task Force for the Management of Arterial Hypertension of the European Society of Hypertension (ESH) and of the European Society of Cardiology (ESC). J Hypertens 2007;25:1105-1187.

4. Chobanian AV, Bakris GL, Black HR, et al. Seventh report of the Joint National Committee on Prevention, Detection, Evaluation, and Treatment of High Blood Pressure. Hypertension 2003;42:1206-1252.

5. Kloke HJ, Branten AJ, Huysmans FT, et al: Antihypertensive treatment of patients with proteinuric renal diseases: risks or benefits of calcium channel blockers? Kidney Int 1998;53:1559-1573.

6. Roggenenti P, Perna A, Loriga G, et al; REIN-2 study group: Blood-pressure control for renoprotection in patients with non-diabetic chronic renal disease (REIN-2): multicentre, randomised controlled trail. Lancet 2005;365:939-946.

7. Bakris GL, Toto RD, McCullough PA, et al; GUARD (gauging albuminuria reduction with Lotrel in diabetic patients with hypertension) study investigators: Effects of different ACE inhibitor combinations on albuminuria: results of the GUARD study. Kidney Int. 2008;73:1303-1309.

8. Hansen PB, Jensen BL, Andreasen D, et al: Differential expression of Tand L-type voltage-dependent calcium channels in renal resistance vessels. Circ Res 2001;89:630-638.

9. Fujita $\mathrm{T}$, Ando $\mathrm{K}$, Nishimura $\mathrm{H}$, et al, on behalf of the Cilnidipine versus Amlodipine Randomized Trial for Evaluation in Renal Disease (CARTER) Study Investigators: Antiproteinuric effect of the calcium channel blocker cilnidipine added to renin-angiotensin inhibition in hypertensive patients with chronic renal disease. Kidney Int 2007;72:1543-1549.

10. Takahara A, Koganei H, Takeda T, et al: Antisympathetic and hemodynamic property of a dual L/N-type Ca2+ channel blocker cilnidipine in rats. Eur J Pharmacol 2002;434:43-47. 
11. Takahara A, Iwasaki H, Nakamura $Y$, et al: Cardiac effects of L/N-type $\mathrm{Ca} 2+$ channel blocker cilnidipine in anesthetized dogs. Eur J Pharmacol 2007;565:166-170.

12. Kishi T, Hirooka $Y$, Konno S, et al:. Cilnidipine inhibits the sympathetic nerve activity and improves baroreflex sensitivity in patients with hypertension. Clin Exp Hypertens. 2009;31:241-249.

13. Zhou $\mathrm{X}$, Ono $\mathrm{H}$, Ono $\mathrm{Y}$, et al: N- and L-type calcium channel antagonist improves glomerular dynamics, reverses severe nephrosclerosis, and inhibits apoptosis and proliferation in an l-NAME/SHR model. J Hypertens 2002;20:993-1000.

14. Konnno Y, Kimura K. Vasodilatory effect of cilnidipine, an L-type and N-type calcium channel blocker, on rat kidney glomerular arteries. Int Heart J 2008;49:723-732.

15. Mena-Martin FJ, Martin-Escudero JC, Simal-Blanco F, et al: Influence of sympathetic activity on blood pressure and vascular damage evaluated by means of urinary albumin excretion. J Clin Hypertens 2006;8:619-624.

16. Krespi PG, Makris TK, Hatzizacharias AN, et al: Moxonidine effect on microalbuminuria, thrombomodulin, and plasminogen activator inhibitor-1 levels in patients with essential hypertension. Cardiovasc Drug Ther 1998;12:463-467.

17. Matsuoka H, Ando $\mathrm{K}$, Ueshima $\mathrm{K}$, et al, on behalf of the SAKURA Trial Investigators. Design and rationale of the Study of Assessment for Kidney function by Urinary microalbumin in RAndomized (SAKURA) trial: Comparison of the antialbuminuric effects between L-/N-type and L-type calcium channel blocker in hypertensive patients with diabetes and microalbuminuria. Clin Exp Hypertens. 2011;33:455-462.

18. Katayama K, Nomura S, Ishikawa H, et al: Comparison between valsartan and valsartan plus cilnidipine in type II diabetics with normo- and microalbuminuria. Kidney Int. 2006;70:151-156.

19. Fogari R, Preti P, Zoppi A, et al: Effects of amlodipine fosinopril combination on microalbuminuria in hypertensive type 2 diabetic patients. Am J Hypertens. 2002;15:1042-1049.

20. The Japanese Society of Nephrology. Clinical Practice Guidebook for Diagnosis and Treatment of Chronic Kidney Disease. 2009. Tokyo: The Japanese Society of Nephrology. 2009. (In Japanese).

21. Matsuo S, Imai E, Horio M, Yasuda Y, et al; Collaborators developing the Japanese equation for estimated GFR. Revised equations for estimated GFR from serum creatinine in Japan. Am J Kidney Dis 2009;53:982-992.

22. Kojima S, Shida M, Yokoyama H. Comparison between cilnidipine and amlodipine besilate with respect to proteinuria in hypertensive patients with renal diseases. Hypertens Res 2004;27:379-385.

23. Morimoto S, Yano Y, Makia K, et al: Renal and vascular protective effects of cilnidipine in patients with essential hypertension. J Hypertens 2007;25:2178-2183

24. Miwa Y, Tsuchihashi T, Ohta $Y$, et al: Antiproteinuric effect of cilnidipine in hypertensive Japanese treated with renin-angiotensin-system inhibitors - A multicenter, open, randomized trial using 24-hour urine collection. Clin Exp Hypertens 2010;32:400-405.

25. Konoshita T, Makino Y, Kimura T, et al; Genomic Disease Outcome Consortium Study Investigators: A new-generation N/L-type calcium channel blocker leads to less activation of the renin-angiotensin system compared with conventional L type calcium channel blocker. J Hypertens 2010;28:2156-2160.

26. Masuo K, Lambert GW, Esler MD, et al: The role of sympathetic nervous activity in renal injury and end-stage renal disease. Hypertens Res. 2010;33:521-528.

27. Weinrauch LA, D'Elia JA, Gleason RE, et al: Autonomic function in type I diabetes mellitus complicated by nephropathy: A cross-sectional analysis presymptomatic phase. Am J Hypertens 1995;8:782-789.

28. Hirschberg R, Kopple JD. The growth hormone-insulin-like growth factor I axis and renal glomerular function. J Am Soc Nephrol 1992;2:1417.

29. Anderson S, Vora JP. Current concepts of renal hemodynamics in diabetes. J Diab Complications 1995;9:304

30. Sabbatini M, Sansone G, Uccello F, et al. Early glycosylation products induce glomerular hyperfiltration in normal rats. Kidney Int 1992;42:875.

31. Vallon V, Blantz RC, Thomson S. Glomerular hyperfiltration and the salt paradox in early type 1 diabetes mellitus: a tubulo-centric view. J Am Soc Nephrol 2003;14:530.

32. Fujisawa T, Ikegami H, Noso S, et al: Renoprotective effect of N-type Ca channel blockade in diabetic nephropathy. J Diab Complications 2007;21:252-257.

33. Masuda T, Ogura MN, Moriya T, et al: Beneficial effects of L- and N-type calcium channel blocker on glucose and lipid metabolism and renal function in patients with hypertension and type II diabetes mellitus. Cardiovasc Ther. 2011;29:46-53.

34. Fukumoto S, Ishimura E, Motoyama K, et al:. Antialbuminuric advantage of cilnidipine compared with L-type calcium channel blockers in type 2 diabetic patients with normoalbuminuria and microalbuminuria. Diab Res Clin Pract 2012, in press.

35. Abe M, Okada K, Maruyama N, et al: Benidipine reduces albuminuria and plasma aldosterone in mild-to-moderate stage chronic kidney disease with albuminuria. Hypertens Res. 2011;34:268-273.

36. Abe M, Okada K, Maruyama N, et al: Comparison between the antiproteinuric effects of the calcium channel blockers benidipine and cilnidipine in combination with angiotensin receptor blockers in hypertensive patients with chronic kidney disease. Expert Opin Investig Drugs. 2010;19:1027-1037.

37. Seino H, Miyaguchi S, Yamazaki T, et al: Effect of benidipine hydrochloride, a long-acting T-type calcium channel blocker, on blood pressure and renal function in hypertensive patients with diabetes mellitus. Analysis after switching from cilnidipine to benidipine. Arzneimittelforschung. 2007;57:526-531.

38. Luippold G, Beilharz M, Muhlbauer B. Chronic renal denervation prevents glomerular hyperfiltartion in diabetic rats. Nephrol Dial Transplant 2004;19:343-347.

39. Strojek K, Grzeszczak W, Górska J, et al: Lowering of microalbuminuria in diabetic patients by a sympathocoplegic agent: novel approach to prevent progression of diabetic nephropathy? J Am Soc Nephrol. 2001;12:602-605.

40. Yokoyama H, Sone H, Oishi M, et al, and on behalf of Japan Diabetes Clinical Data Management Study Group: Prevalence of albuminuria and renal insufficiency and associated clinical factors in type 2 diabetes: the Japan Diabetes Clinical Data Management study (JDDM15). Nephrol. Dial. Transplant. 2009;24:1212-1219.

41. Afghahi H, Cederholm J, Eliasson B, et al: Risk factors for the development of albuminuria and renal impairment in type 2 diabetes-the Swedish National Diabetes Register (NDR). Nephrol. Dial. Transplant. 2011;26:1236-1243. 